\title{
Cherenkov friction on a neutral particle moving parallel to a dielectric
}

\author{
Gregor Pieplow and Carsten Henkel \\ Institute of Physics and Astronomy, Universität Potsdam, Germany
}

18 Feb 2014

\begin{abstract}
Based on a fully relativistic framework and the assumption of local equilibrium, we describe a simple mechanism of quantum friction for a particle moving parallel to a dielectric. The Cherenkov effect explains how the bare ground state becomes globally unstable and how fluctuations of the electromagnetic field and the particle's dipole are converted into pairs of excitations. Modelling the particle as a silver nano-sphere, we investigate the spectrum of the force and its velocity dependence. We find that the damping of the plasmon resonance in the silver particle has a relatively strong impact near the Cherenkov threshold velocity. We also present an expansion of the friction force near the threshold velocity for both damped and undamped particles.
\end{abstract}

Key words: radiation force, quantum friction, Cherenkov, quantum fluctuations. - PACS: 12.20.-m, 42.50.Lc, 05.30.-d, 03.65.-w, 03.50.De, 42.50.Wk, 03.30.+p

\section{Introduction}

The conversion of mechanical energy into heat is referred to as friction in most cases. Numerous mechanisms can be identified that cause friction, but it is still a challenge to infer macroscopic observations from microscopic phenomena. So far only very simple scenarios permit a detailed analysis of the fundamental aspects of friction. A prominent example is the theory of the quantized electromagnetic field applied to the case of two parallel moving plates separated by a small vacuum gap [1-5]; see Refs. [6-9] for reviews. Friction arises due to the spontaneous creation of particle pairs that propagate away into the plates or are dissipated there. A similar treatment can 
be applied to a body moving above a flat surface at constant speed [10-13]. Taking advantage of Lorentz invariance, one achieves treatments consistent with special relativity [14,15], as required for the archetypal situation that high-energy charges are stopped in a medium. In a recent paper, we described such a formalism for a neutral, polarizable particle moving parallel to a flat interface [16]. At a typical distance of at least a few nanometers (larger than the atomic scale), the interaction depends on a few macroscopic parameters (refractive index, conductivity, surface impedance ...). In the present paper we discuss a special configuration of this setting with a dielectric medium below the surface, and with both particle and medium at zero temperature. These conditions make the friction a pure quantum-mechanical drag and closely relates it to the realm of Casimir phenomena [17]. A friction force appears when the speed of the particle relative to the surface exceeds the velocity of light in the medium $(c / n)$ : this drag can thus be attributed to the Cherenkov effect. The situation is somewhat unusual because neither the surface nor the particle have to be dissipative. All that is required are spectral mode densities for the medium field and the particle. For a moving charge the Cherenkov drag is well known and is described easily with classical electromagnetic theory [18]. A neutral body requires a more refined treatment, as quantum fluctuations have to be treated accordingly. As in previous work [3, 19], we use the fluctuation-dissipation theorem to do just that. Because of the growing interest in this field and some controversy surrounding it (see Ref. [13] for a review), the simple situation studied here might provide another test bed to compare current results and ideas in detail.

In this paper, we analyze in detail a spectral representation of the friction force that must be applied to move a small particle parallel to a flat dielectric surface. While this setup has obvious applications for micro- and nano-machines, our focus is on illustrating the underlying mechanisms. The basic physics is very similar to the seminal explanation of the Cherenkov effect [20] by Tamm and Frank [21]: for a certain sector of field modes, the Doppler shift flips the sign of the mode frequency (anomalous Doppler effect). This leads to scattering relations (Smatrix) in the form of a Bogoliubov transformation [7.9]: incident waves get amplified, and pairs of elementary excitations (photon-polaritons) can be created out of the quantum fluctuations in the field and in the particle's dipole moment. The frictional force arises from the power carried away by these excitations as they are absorbed or as they propagate into the bulk of the body. The recent paper by Barton [13] provides a particularly transparent calculation of these processes in a simplified setting (only surface plasmon modes are considered). The starting point we use here is based on the fluctuation electrodynamics developed by Rytov and co-workers [19]: the basic assumption is that both the solid surface and the moving particle are in local thermodynamic equilibrium. This is a good approximation for a mesoscopic particle made from thousands of atoms, at least over time scales where its temperature can be considered constant (large heat 
capacity). The approximation is much more questionable for microscopic particles like atoms or molecules because these may settle into a non-thermal state due to spontaneous excitation.

We structure our analysis in the following way: some results of previous work are summoned to provide the basis for the Cherenkov effect. The quantum (Cherenkov) friction is then calculated and its physical properties are discussed. We link the friction force to an absorbed power that has to be provided to move the particle at constant speed. A relativistic argument put forward by Polevoi [3] attributes this power to an increase in mass-energy. After the analytics, we numerically investigate a silver nano-particle moving at relativistic speed above a dielectric surface. We quantify the magnitude of the friction, and provide a geometric picture of most of the features that determine the friction spectrum. We then present an expansion of frequency spectrum of the force and of the force itself near the threshold in $(v-c / n)$. This further illustrates the relative importance of the resonance and the low, off-resonant frequencies in the particle polarizability. We find a remarkable agreement with the numerical results close to the threshold. The main result is that the Cherenkov friction is linked to composite modes at the vacuum-dielectric interface [22,23] which couple to the particle via their evanescent vacuum tail; their plane-wave component in the medium can be seen as carrying away the dissipated power.

\section{The formalism}

\subsection{Friction force}

In an earlier paper [16] we presented a covariant approach to the force on a particle that moves with arbitrary speed parallel to a flat surface that responds linearly to electromagnetic waves. We recovered the results of Refs. [14,24]. The formalism allows for different temperatures of particle and surface, assuming a state of local equilibrium. The relative motion leads to Doppler shifts that are handled by Lorentz transforming an incident field into the frame co-moving with the particle or the surface. The Doppler-shifted frequency distribution of the equilibrium distributions are responsible for a non-equilibrium force that persists even when both temperatures $T \rightarrow 0$.

Let us fix coordinates such that the $x$-axis points along the motion of the particle (velocity v), while the half-space $z \leq 0$ coincides with the medium. According to Refs. [14, 16], the force component $F_{x}$ acting on the particle (at distance $z$ from the surface) is

$$
F_{x}=\frac{\hbar}{2 \gamma} \int \frac{\mathrm{d} \omega}{2 \pi} \frac{\mathrm{d}^{2} k_{\|}}{(2 \pi)^{2}}\left[\operatorname{sign}(\omega)-\operatorname{sign}\left(\omega-v k_{x}\right)\right] \times
$$




$$
k_{x} \operatorname{Im} \alpha\left[\gamma\left(\omega-v k_{x}\right)\right] \sum_{\sigma=s, p} \phi_{\sigma}\left(\omega, \mathbf{k}_{\|}\right) \operatorname{Im}\left(\frac{r_{\sigma} \mathrm{e}^{-2 \kappa z}}{\kappa}\right) .
$$

The frequency $\omega$ and parallel wave numbers $\mathbf{k}_{\|}=\left(k_{x}, k_{y}\right)$ are measured in the rest frame of the medium; the integral boundaries are $(-\infty, \infty)$. The difference of sign functions arises from the thermal factors $\operatorname{coth}\left[\hbar \omega /\left(2 k_{\mathrm{B}} T\right)\right]$ in the zero-temperature limit, evaluated in the respective rest frames of medium and particle $\left(\omega^{\prime}=\gamma\left(\omega-v k_{x}\right)\right)$. The particle polarizability is $\alpha, \gamma$ is the Lorentz factor, and for the weight functions $\phi_{\sigma}$ we have (setting $c=1$ )

$$
\begin{aligned}
& \phi_{s}\left(\omega, \mathbf{k}_{\|}\right)=\omega^{2}+2 \gamma^{2}\left(\mathbf{v} \times \mathbf{k}_{\|}\right)^{2}\left(1-\frac{\omega^{2}}{k_{\|}^{2}}\right), \\
& \phi_{p}\left(\omega, \mathbf{k}_{\|}\right)=\omega^{\prime 2}+2 \gamma^{2}\left(k_{\|}^{2}-\left(\mathbf{v} \cdot \mathbf{k}_{\|}\right)^{2}\right)\left(1-\frac{\omega^{2}}{k_{\|}^{2}}\right) .
\end{aligned}
$$

The reflection coefficients for $p$ - and $s$-polarized light are

$$
r_{\mathrm{s}}=\frac{\mathrm{i} \kappa-\kappa_{n}}{\mathrm{i} \kappa+\kappa_{n}} \quad, \quad r_{\mathrm{p}}=\frac{\mathrm{i} n^{2} \kappa-\kappa_{n}}{\mathrm{i} n^{2} \kappa+\kappa_{n}}
$$

where $\kappa=\sqrt{k_{\|}^{2}-(\omega+i 0)^{2}}$ and $\kappa_{n}=\sqrt{n^{2}(\omega+i 0)^{2}-k_{\|}^{2}}$. Here, $n$ is the refractive index of the medium.

Using symmetries and other properties we can further simplify the integral in Eq.(1). The integrand is even under the transformations $\left(\omega, k_{x}\right) \mapsto\left(-\omega,-k_{x}\right)$ and $k_{y} \mapsto-k_{y}$ so that it is sufficient to integrate over the domain $\omega>0, k_{y}>0$. The difference of the sign-functions reduces to a factor of two for $0<\omega<v k_{x}$. This wedge-shaped domain in the $k_{x}, \omega$-plane is below the projected light cone $\omega=k_{\|}$, so that only fields that are evanescent at the particle's location contribute to the force [Fig, 1 (left)]. We conclude that the factor $\mathrm{e}^{-2 \kappa z} / \kappa$ is real-valued.

Another crucial insight is contained in the reflection coefficients (4): their imaginary part is nonzero only in the annulus $\omega<k_{\|}<n \omega$ [see Fig.2(right) below]. With the condition derived from the sign functions we get $\omega<v k_{\|} \cos \phi<v n \omega \cos \phi$, so that the condition for Cherenkov radiation follows

$$
1<v n \cos \phi
$$

where $\phi$ is the angle between $\mathbf{v}$ and $\mathbf{k}_{\|}$. The expression (1) for the force thus becomes:

$$
\begin{aligned}
F_{x}= & \frac{4 \hbar}{\gamma(2 \pi)^{3}} \int_{0}^{\infty} \mathrm{d} \omega \int_{\omega / v}^{n \omega} \mathrm{d} k_{x} \int_{0}^{\sqrt{n^{2} \omega^{2}-k_{x}^{2}}} \mathrm{~d} k_{y} \\
& k_{x} \operatorname{Im} \alpha\left[\gamma\left(\omega-v k_{x}\right)\right] \sum_{\sigma=s, p} \phi_{\sigma}\left(\omega, \mathbf{k}_{\|}\right) \operatorname{Im}\left(r_{\sigma}\right) \frac{\mathrm{e}^{-2 \kappa z}}{\kappa} .
\end{aligned}
$$



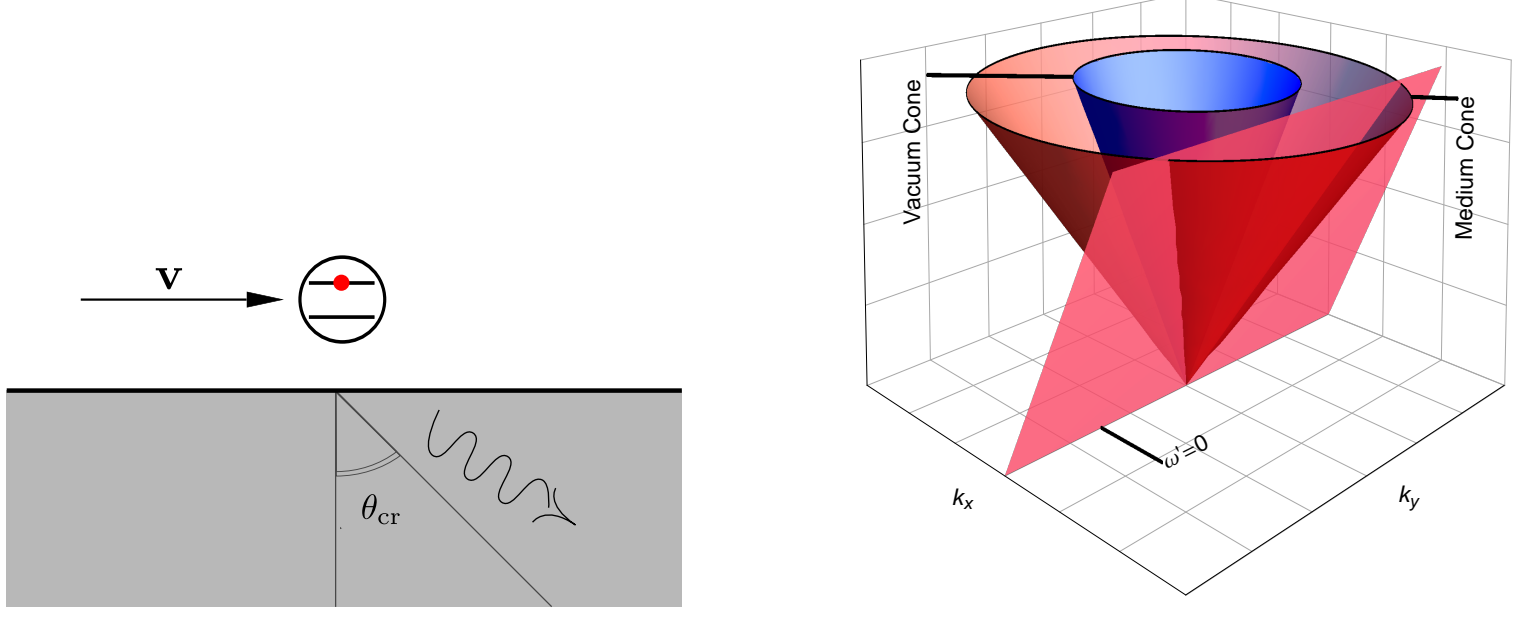

Figure 1. Sketch of the system and visualization of the relevant photon modes.

(left) Kinematics of Cherenkov (quantum) friction: the moving particle is spontaneously excited and a photon is emitted into the medium beyond the critical angle. (right) Cherenkov friction arises from a domain in the $\omega, \mathbf{k}_{\|}$-space that is enclosed by the projected light cone in the medium $\omega=k_{\|} / n$ (dark red) and the plane $\omega=v k_{x}$ (pink). Below this plane, the Doppler shift is anomalous and in the frame moving with the particle $\omega^{\prime}<0$. All points below the vacuum light cone (blue) correspond to evanescent waves bound to the medium surface. We take $n=2$ and $v=0.8 c>c / n$.

\subsection{Photon emission and anomalous Doppler shift}

The manipulations performed so far have a clear physical meaning within the theory of the Cherenkov effect [18, 20,21] which is well understood. A kinematic explanation of the friction above the Cherenkov threshold can be given following the equations outlined in Ref. [25]. We start with the conservation of 4-momentum

$$
p_{1}^{\mu}=\hbar k^{\mu}+p_{2}^{\mu}
$$

The momenta $p_{a}^{\nu}$ describe the particle before and after the emission of a photon with momentum $\hbar k^{\nu}$, where $a=1,2$ labels the internal states (energy levels $\epsilon_{1,2}$ ). Although Eq.(7) and Ref. [25] deal with a particle moving through a medium, the physics is the same for the motion parallel to the dielectric medium. We have for the particle and the photon (recall that $c=1$ )

$$
p_{a}^{\mu}=\left(E_{a}, \gamma m_{a} \mathbf{v}\right), \quad m_{a}=M+\epsilon_{a},
$$




$$
\begin{aligned}
E_{a} & =\sqrt{m_{a}^{2}+\gamma^{2} m_{a}^{2} \mathbf{v}^{2}}=\gamma m_{a} \\
k^{\mu} & =(\omega, \mathbf{k}), \quad k=n \omega .
\end{aligned}
$$

The Greek indices run from 0 to 3, and toggling between co- and contravariant indices is done with the metric $g_{\mu \nu}=\operatorname{diag}(1,-1,-1,-1)$. It is understood that $k=\sqrt{\mathbf{k}^{2}}$. The masses $m_{a}$ are associated with the particle's energy levels. The photon is supposed to be emitted into the medium, hence the dispersion relation in eq.(10). Because the particle is pushed by an "invisible hand", the velocity $\mathbf{v}$ does not change during the emission. This is equivalent to neglecting the recoil [25] of the particle. Squaring eq. (7) leads to

$$
\left(\epsilon_{1}-\epsilon_{2}\right)\left(2 M+\epsilon_{1}+\epsilon_{2}\right)=2 E_{1} \hbar \omega(1-v n \cos \phi) \text {. }
$$

with the same notation as in Eq. (5) above. We can reasonably make the approximation $\epsilon_{1,2} \ll M$ so that we recover

$$
\hbar \omega=-\frac{\epsilon_{2}-\epsilon_{1}}{\gamma(1-v n \cos \phi)} .
$$

If the particle is faster than the speed of light inside the medium, $1 / n$, the denominator is negative [Cherenkov condition (5)]. This is an illustration of the so-called anomalous Doppler effect where the photon frequency, as seen from the moving particle, $\omega^{\prime}=\gamma\left(\omega-v k_{x}\right)$, is negative. The authors of Ref. [25] point out that this allows for the excitation of the particle to a higher energy level, $\epsilon_{2}>\epsilon_{1}$, while emitting a photon into the medium, inside the Cherenkov cone [see Fig[1(left)]. The power lost into the emission must be supplied by the force that keeps the particle on its track. In other words, considering quantum electrodynamics at a dielectric interface coupled to a polarizable particle moving faster than the Cherenkov threshold, it turns out that this is an example of an unstable field theory [7, 26], similar to electron-positron production in strong electric fields and Hawking radiation in a strong gravitational field.

\subsection{Heating and frictional power}

This simple kinematic analysis corresponds neatly to the integration domain in eqs.(1, 6). Note in particular that the particle's response function is evaluated at the Doppler-shifted frequency and yields $\operatorname{Im} \alpha\left(\gamma\left(\omega-v k_{x}\right)\right)<0$ in the domain. This is a clear indicator that the anomalous Doppler effect in combination with the photon emission of photons into the Cherenkov cone indeed slows down the particle. Another quantity of interest is the rate of mass change in the particle's comoving frame. This is given by $\dot{m}=u^{\mu} F_{\mu}$ where $u_{\mu}$ is the particle's 4-velocity. The full 4-vector 
of force $F_{\mu}$ can be found in [16], and for our particle moving in the $x$-direction, we find

$$
\begin{aligned}
\dot{m} & =\gamma\left(F_{0}-v F_{x}\right) \\
\left(\begin{array}{c}
F_{0} \\
v F_{x}
\end{array}\right) & =\int_{0}^{\infty} \mathrm{d} \omega \int_{\omega / v}^{n \omega} \mathrm{d} k_{x} \int_{0}^{\sqrt{n^{2} \omega^{2}-k_{x}^{2}}} \mathrm{~d} k_{y}\left(\begin{array}{c}
-\hbar \omega \\
-\hbar v k_{x}
\end{array}\right) \Gamma\left(\omega, \mathbf{k}_{\|}\right)
\end{aligned}
$$

where the positive quantity

$$
\Gamma\left(\omega, \mathbf{k}_{\|}\right)=\frac{4}{\gamma(2 \pi)^{3}} \operatorname{Im} \alpha\left[\gamma\left(v k_{v}-\omega\right)\right] \sum_{\sigma=s, p} \phi_{\sigma}\left(\omega, \mathbf{k}_{\|}\right) \operatorname{Im}\left(r_{\sigma}\right) \frac{\mathrm{e}^{-2 \kappa z}}{\kappa}
$$

can be identified as a spectrally resolved photon emission rate. (We exploited the fact that $\operatorname{Im} \alpha\left(\omega^{\prime}\right)$ is an odd function.) Note that the proper mass increases, $\dot{m}>0$, because per emission event, a positive energy $-\hbar \omega^{\prime}=\hbar \gamma\left(v k_{x}-\omega\right)$ is dumped into the particle's internal mass-energy, as discussed in the previous section. Indeed, we shall see, through a simple oscillator model for the polarizability, that the frequency $\omega^{\prime}$ in the co-moving frame is essentially fixed by the particle's resonance.

To summarize this section, let us re-write the power balance as a sum of two positive terms:

$$
-v F_{x}=-F_{0}+\frac{\mathrm{d} m}{\gamma \mathrm{d} \tau}
$$

On the left-hand side, we see the frictional power spent to maintain the constant speed of the particle. The first term on the right-hand side gives the power of photon emission (energy $\hbar \omega$ at rate $\Gamma\left(\omega, \mathbf{k}_{\|}\right)$, see Eq. 14 ), while the second gives the power absorbed in the particle. (The factor $1 / \gamma$ gives the relativistic time dilation between the particle's proper time $\tau$ and the laboratory time t.)

\section{Case study: relativistic nanoparticle}

\subsection{Numerical investigations}

To illustrate further the physical features of the Cherenkov friction force, we provide some numerical estimates for a metallic nano-particle. We chose a silver nano-sphere with radius $a=3 \mathrm{~nm}$ that moves at a distance $z=10 \mathrm{~nm}$ above a dielectric medium with refractive index $n=2$. For simplicity, frequency dispersion is neglected in the medium [23]. For the particle, we adopt a Drude model with parameters for silver: plasma frequency $\hbar \omega_{\mathrm{pl}}=9.01 \mathrm{eV}$ and damping rate $\hbar / \tau=16 \mathrm{meV}$ (not to be confused with the proper time coordinate $\tau$ above). For such a small 
particle, the first term of the Mie series will suffice so that its response is given by the electric dipole polarizability (for SI units, multiply with the Coulomb constant $\varepsilon_{0}$ )

$$
\alpha(\omega)=4 \pi a^{3} \frac{\varepsilon(\omega)-1}{\varepsilon(\omega)+2}=4 \pi a^{3} \frac{\Omega^{2}}{\Omega^{2}-\omega^{2}-\mathrm{i} \omega / \tau}
$$

where $\varepsilon(\omega)$ is the metal permittivity. The resonance at $\Omega=\omega_{\mathrm{pl}} / \sqrt{3}$ corresponds to a plasmon mode localized on the particle. The calculations simplify considerably in the no-damping limit $\tau \rightarrow \infty$ which gives

$$
\lim _{\tau \rightarrow \infty} \operatorname{Im} \alpha(\omega)=2 \pi^{2} a^{3} \omega[\delta(\omega-\Omega)+\delta(\omega+\Omega)] .
$$

We have checked that at this distance and for velocities above the Cherenkov threshold, both polarizations contribute roughly the same amount to the force. This is at variance with the more familiar regime of short (non-retarded) distances and slow (non-relativistic) atoms where the ppolarization dominates and an electrostatic calculation suffices.

Fig.1 (right) above illustrates the simple appearance of the integration volume, which determines most of the features of the force spectrum: it lies between zero-frequency plane $\omega^{\prime}=0$ and the medium light cone $\omega=k_{\|} / n$. The opening angle $\phi_{\max }$ of the intersection (measured in the $\mathbf{k}_{\|}$-plane, relative to the direction of the velocity $\mathbf{v}$ ) is given by the Cherenkov formula

$$
\cos \phi_{\max }=\frac{\omega / v}{n \omega}=\frac{1}{n v}
$$

where $v$ is the particle velocity (scaled to $c$ ).

Fig 2.1 (left) shows the impact of the particle's plasmon resonance: the plane $\omega^{\prime}=-\Omega$ and the medium light cone intersect in a hyperbola whose opening angle (projected onto the $\mathbf{k}_{\| \text {-plane) }}$ is again given by the Cherenkov formula (19) - the higher the speed of the particle, the more inclined the plane. The integrand roughly peaks near the apex of the hyperbola whose position is easily calculated to be

$$
\omega_{\mathrm{a}}=\frac{\Omega}{\gamma(n v-1)}, \quad k_{x \mathrm{a}}=\frac{n \Omega / c}{\gamma(n v-1)}, \quad k_{y \mathrm{a}}=0 .
$$

In Fig 2 (right), we plot a slice at constant frequency through the spectral density $F_{x}\left(\omega, \mathbf{k}_{\|}\right)$of the friction force given by

$$
F_{x}\left(\omega, \mathbf{k}_{\|}\right)=\frac{4 \hbar k_{x}}{\gamma(2 \pi)^{3}} \operatorname{Im} \alpha\left[\gamma\left(\omega-v k_{x}\right)\right] \mathrm{e}^{-2 \kappa z} \sum_{\sigma=s, p} \frac{2 k_{\|} q_{\sigma} \phi_{\sigma}\left(\omega, \mathbf{k}_{\|}\right)}{q_{\sigma}^{2} \kappa^{2}+k_{\|}^{2}}
$$




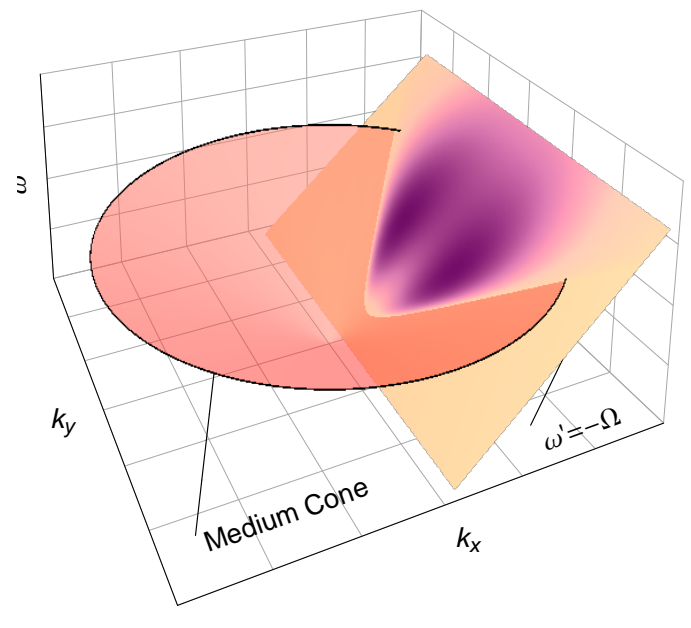

Figure 2. Impact of the particle plasmon resonance.

(left) Spectral density $F_{x}\left(\omega, \mathbf{k}_{\|}\right)$of the friction force plotted in the plane $\omega^{\prime}=-\Omega$ where the particle's oscillator strength $\operatorname{Im} \alpha\left(\omega^{\prime}\right)$ peaks. The inclination of the plane is determined by the velocity of the particle. The intersection with the medium light cone (pink) is a hyperbola whose apex has the coordinates given in Eq. 20].

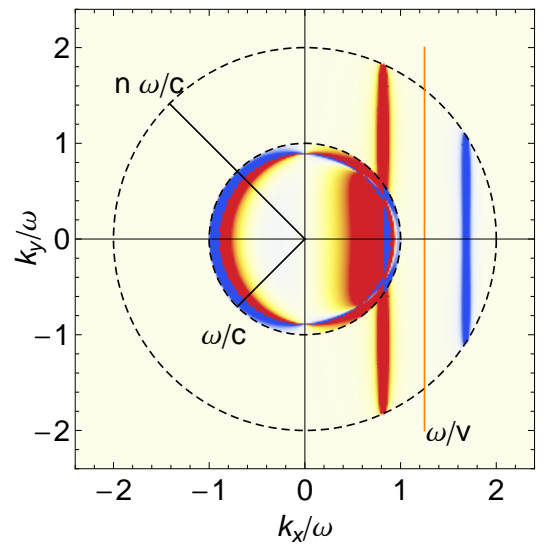

(right) Density plot of $F_{x}\left(\omega, \mathbf{k}_{\|}\right)$in the $k_{x}, k_{y}$-plane (cut through the left figure at constant frequency). The dashed lines give the outline of the light cones in vacuum and the medium. The relevant integration domain is inside the larger circle and to the right of the orange line $k_{x}=\omega / v$. The wide blue line in this area illustrates the absorption resonance $\omega^{\prime}=-\Omega$ of the particle polarizability $\alpha\left(\omega^{\prime}\right)$. If $T \neq 0$, the orange line blurs, and the inner circle contributes to the integral as well. Red/blue colors represent positive/negative values. Parameters: $\omega=\sqrt{3} \Omega$, where $\Omega$ is the particle's resonance. We took a relatively short damping time with $\Omega \tau=32.5$.

where the imaginary part of the reflection amplitudes $r_{\sigma}$ was worked out from Eqs.(4), and $q_{s}=1$ and $q_{p}=n^{2}$. The density plot reveals how the resonances of the polarizability $\alpha\left(\omega^{\prime}\right)$ select narrow stripes in the $\mathbf{k}_{\|}$plane. Only the resonance $\omega^{\prime}=-\Omega$ (blue) lies in the integration domain relevant for quantum friction.

These geometric considerations carry over when we integrate over $k_{x}$ and $k_{y}$ and consider the force spectrum. This is illustrated in Fig.3. Photon emission resonant with the particle plasmon resonance becomes dominant at velocities well above the Cherenkov threshold [Fig 3 (left)]. Closer to the threshold, contributions at lower frequency arise from photons that are off-resonant, more precisely quasi-static, in the frame co-moving with the particle. Similar to Cherenkov radiation, they are boosted into the visible range by the Doppler shift. These photons arise from the 
nonzero value of the polarizability at low frequencies

$$
\omega^{\prime} \ll \Omega: \quad \operatorname{Im} \alpha\left(\omega^{\prime}\right) \approx 4 \pi a^{3} \frac{\omega^{\prime}}{\Omega^{2} \tau}
$$

Note that the only material parameter in this regime is the metal conductivity $\sigma=\varepsilon_{0} \Omega^{2} \tau$, see also Refs. [4, 27]. Our interpretation is confirmed in Fig 3 (right) where the spectrum is also calculated in the lossless limit, using the approximate polarizability (18). Off-resonant photon emission is suppressed, and the frequency $\omega_{\mathrm{a}}$ from Eq. 20) provides a sharp threshold.

Finally, the total friction force is plotted as a function of the particle velocity in Fig.4 Note again the relatively large difference between finite damping and the lossless limit near the Cherenkov threshold.
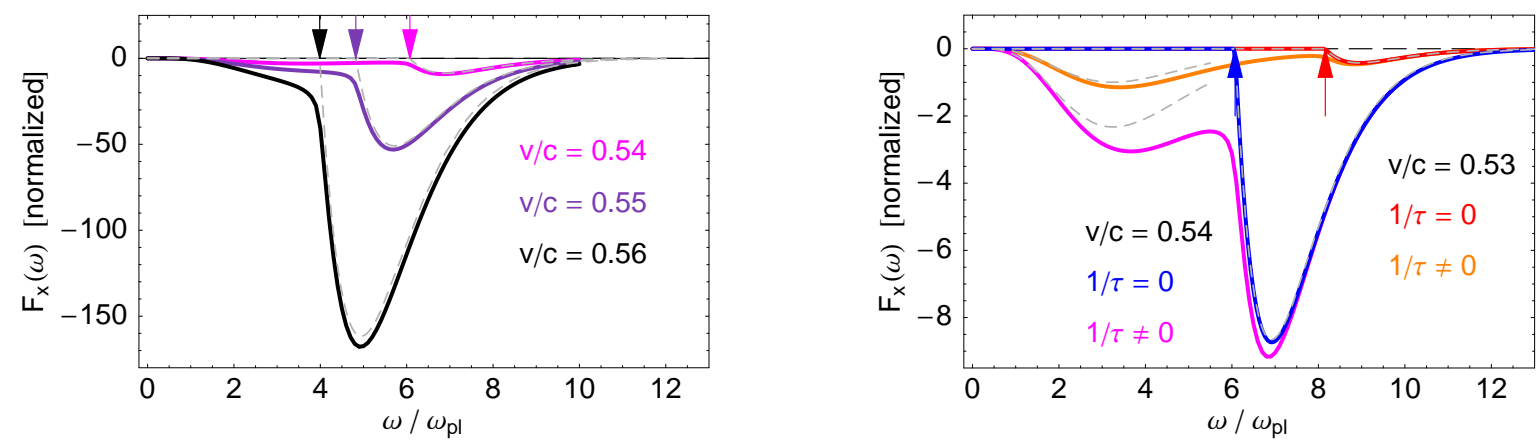

Figure 3. Impact of particle velocity and plasmon damping on quantum friction.

(left) Frequency spectrum $F_{x}(\omega)$ of the friction force for a silver nano-particle at different velocities above the Cherenkov threshold $c / n=0.5$, obtained by integrating $F_{x}\left(\omega, \mathbf{k}_{\|}\right)$over $\mathbf{k}_{\|}$. The arrows give the apex of the hyperbola [Eq.[20p] shown in the left plot. We used the quite arbitrary normalization factor $4 \hbar\left(4 \pi a^{3}\right)(2 \pi)^{-3} 10^{-4}\left(\omega_{\mathrm{pl}} / c\right)^{4} \approx 3.4 \mathrm{aN} / \omega_{\mathrm{pl}}$ for the force spectrum. We took a damping time fixed by $\Omega \tau=$ 32.5 , which is shorter than in a bulk due to electron scattering at the nano particle surface [28-31]. (right) Comparison of the lossless case $1 / \tau=0$ and a particle resonance with a finite width (same parameters as in Fig 2). The arrow indicates the frequency $\omega_{\mathrm{a}}$ [Eq.20]] where the particle resonance $\omega^{\prime}=-\Omega$ intersects the light cone in the medium (apex of the hyperbola in Fig 2.1 (left)). Same normalization as in Fig 3 (left).

\subsection{Approximations near threshold}

The integrals can be calculated approximately when the opening angle of the Cherenkov cone is very narrow $(v \approx 1 / n)$. The main features are captured by the reflection coefficient in $\mathrm{p}$ polarization, expanded for small $\kappa_{n}$ [see Eq.(4)]. (See the Appendix for more details.) The 
formulas of this section are represented in dashed (gray) lines on Figs, 3, 4, the agreement is quite remarkable.

For a particle polarizability with a very narrow resonance, we find the approximate spectrum

$$
\begin{aligned}
\omega \geq \omega_{\mathrm{a}}: \quad F_{x}(\omega) \mathrm{d} \omega & \approx-\frac{4 \hbar\left(4 \pi a^{3}\right)}{(2 \pi)^{3}} \frac{\pi^{2} \Omega}{8 n v \gamma^{2}} \mathrm{~d} \omega \omega k_{y \max }^{2} \mathrm{e}^{-2 \omega z \sqrt{n^{2}-1}} \times \\
\times & \left(4+\frac{3 k_{y \max }^{2} z}{\omega \sqrt{n^{2}-1}}\right) \\
k_{y \max }^{2} & =(n v-1) \frac{\omega-\omega_{\mathrm{a}}}{v^{2}}\left[2 \omega+(n v-1)\left(\omega+\omega_{\mathrm{a}}\right)\right]
\end{aligned}
$$

where $\omega_{\mathrm{a}}$ is given by Eq. (20), and $k_{y \max }$ parametrizes the width of the hyperbola in Fig.2(left). This spectrum has a sharp threshold (dashed gray lines in Figs 3). If the polarizability includes damping, the contribution from quasi-static frequencies can be computed similarly, using the approximation (22). The resulting spectrum is

$$
\begin{aligned}
\omega \sim 0: \quad F_{x}(\omega) \mathrm{d} \omega \approx-\frac{4 \hbar}{(}\left(4 \pi a^{3}\right) & \frac{\pi(n v-1)^{3}}{n^{2} v^{6} \Omega^{2} \tau} \mathrm{d} \omega \omega^{5} \mathrm{e}^{-2 \omega z \sqrt{n^{2}-1}} \\
& \times\left(\frac{v^{2}}{20}+\frac{3 n v^{3}}{20}+\frac{2 n^{2} v^{4}}{15}\right. \\
& \left.+\frac{(n v-1) \omega z}{280 \sqrt{n^{2}-1}}\left(5+20 n v+29 n^{2} v^{2}+16 n^{3} v^{3}\right)\right)
\end{aligned}
$$

and peaks roughly at the inverse roundtrip time $1 /\left(z \sqrt{n^{2}-1}\right)$ (dashed lines in Fig. 3 (right)). As illustrated in the figure above, this approximation becomes quite poor away from the threshold, as frequencies above the validity of the low-frequency approximation $(22)$ for $\operatorname{Im} \alpha\left(\omega^{\prime}\right)$ become relevant.

From both approximations for the spectra, the velocity-dependent friction force can be calculated, leading to:

$$
\text { no damping : } \quad \begin{aligned}
F_{x} \approx & -\frac{4 \hbar\left(4 \pi a^{3}\right)}{(2 \pi)^{3}} \frac{\pi^{2} n^{3}}{8\left(n^{2}-1\right)^{3 / 2}} \frac{\omega_{\mathrm{a}}}{z^{4}}(v-1 / n)^{2} \mathrm{e}^{-2 \sqrt{n^{2}-1}} \omega_{\mathrm{a}} z \\
& \times\left(3+4 \sqrt{n^{2}-1} \omega_{\mathrm{a}} z+2\left(n^{2}-1\right)\left(\omega_{\mathrm{a}} z\right)^{2}\right) \\
\text { with damping : } \quad F_{x} \approx & -\frac{4 \hbar\left(4 \pi a^{3}\right)}{(2 \pi)^{3}} \frac{5 \pi n^{5}}{8\left(n^{2}-1\right)^{3}} \frac{(v-1 / n)^{3}}{z^{6} \Omega^{2} \tau} \\
& \times\left(1+(v-1 / n) \frac{11 n-2 n^{3}}{4\left(n^{2}-1\right)}+\mathcal{O}(v-1 / n)^{5}\right)
\end{aligned}
$$


In both cases, we have simplified the complicated polynomial in $v$ to the lowest order above $1 / n$. The dependence on the threshold frequency $\omega_{\mathrm{a}} \sim(v-1 / n)^{-1}$ makes the no-damping result exponentially small at threshold, while damping leads to a cubic power law $\sim(v-1 / n)^{3}$. We also emphasize the different power laws with distance $z$ from the surface; the corrections to the no-damping case in Eq. (26) are quite significant for our parameters, as we have the relatively large value $\omega_{\mathrm{a}} z \approx 2.2$ at $v=0.55 \mathrm{c}$. The numerical calculation for a particle with damping agrees quite well with formula (27) close to the threshold velocity. Around $v \sim 0.53$, the contribution from the resonance takes over and the dependence on the damping constant become negligible.

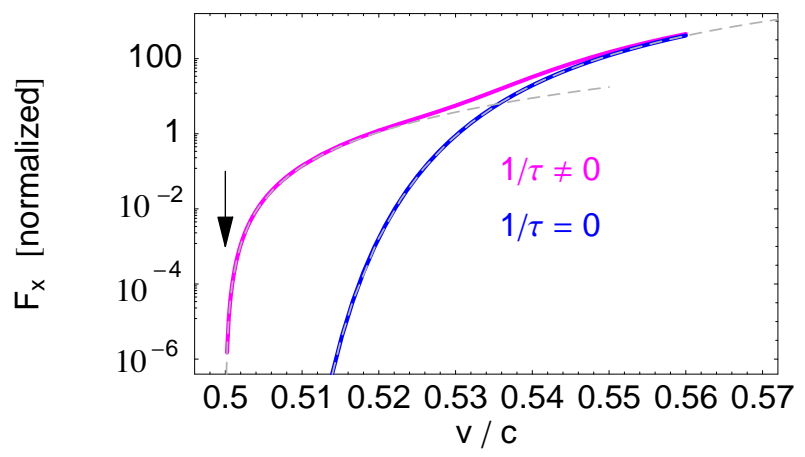

Figure 4: Total friction force vs. particle velocity (log scale). The arrow indicates the Cherenkov threshold $v=c / n$. Similar to Fig, 3 , the force is normalized to the value $\left(2 / \pi^{2}\right) 10^{-4} \hbar\left(\omega_{\mathrm{pl}}^{5} / c^{4}\right) a^{3}$, i.e., an acceleration of $\approx 2900 \mathrm{~m} / \mathrm{s}^{2}$.

\section{Conclusion}

We investigated a neutral particle moving in close proximity parallel to a dielectric. Studying the expression (1) that was derived from a fully relativistic extension of the fluctuation-dissipation theorem, we provided a connection to a fundamental and simple friction mechanism. If the particle moves faster than the speed of light inside the medium (Cherenkov condition), it can dissipate energy by creating pairs of excitations. Unlike as in Ref. [13], the pairs are excitations of the particle and photon modes propagating in the medium. These modes change the sign of their frequency under the Doppler shift (anomalous Doppler effect [21,32]). This leads to an S-matrix in the form of a Bogoliubov transformation that spontaneously excites the particle and generates a photon emitted into the medium [7, 9, 25]. The mechanism we described is 
another example of an unstable vacuum state in a quantum field theory [26]. The main features of Cherenkov friction were explained in geometrical terms by analyzing the frequency spectrum of the force. In order to provide a concrete example, we considered a metallic nano particle whose polarizability is dominated by a plasmon resonance. We found a remarkable agreement of the numerical data with an expansion of the force and the force spectrum in $(v-c / n)$ near the threshold. The approximate expressions further illustrated the role of the low frequency behavior of the particles polarizability and its plasmon resonance.

In order to connect with the current discussion on quantum friction [5, 12, 13, 33], we note that in its simplest form, Cherenkov friction does not require damping in either the particle or the surface. We studied the impact of dissipation in the particle (as described by a damped plasmon mode) and found that this significantly changes the friction force just above the Cherenkov velocity, while maintaining strictly zero friction below the threshold. This changes, however, when finite temperatures are introduced, or absorption is allowed for in the surface. Our general result for the radiative force is identical to that of Ref. [15]. The general setting for the field quantization (lossless and non-dispersive dielectric) is the same as in Ref. [23], however, a different particle is considered there (self-energy of a moving electron). The approach of Ref. [34] is limited to friction forces linear in the relative velocity of two systems which are both at the same temperature. The vanishing of linear friction at $T=0$ is consistent with our analysis. The investigation in Ref. [13] uses a different model for the particle's polarizability: a microscopic two-level system with radiative damping only. In the description of the field modes near the surface, damping (absorption) is allowed for, and only electrostatic fields are considered (non-relativistic limit). We emphasize in particular that the excitations that lead to frictional losses are pairs of surface plasmons in Ref. [13]. A comprehensive picture where the weight of this excitation process can be compared directly to the spontaneous particle excitation studied here still needs to be developed. The simple setting put forward in this paper may provide a route towards such a picture.

\section{Acknowledgements}

We thank V. E. Mkrtchian, H. R. Haakh, and J. Schiefele for helpful discussions in various stages of this work. 


\section{Appendix}

For the sake of convenience we repeat the general expression for the force at $T=0$ :

$$
\begin{aligned}
F_{x}=\frac{4 \hbar}{\gamma(2 \pi)^{3}} \int_{0}^{\infty} \mathrm{d} \omega \int_{\omega / v}^{n \omega} \mathrm{d} k_{x} \int_{0}^{\sqrt{n^{2} \omega^{2}-k_{x}^{2}}} \mathrm{~d} k_{y} \\
\quad \times k_{x} \operatorname{Im} \alpha\left[\gamma\left(\omega-v k_{x}\right)\right] \sum_{\sigma=s, p} \phi_{\sigma}\left(\omega, \mathbf{k}_{\|}\right) \operatorname{Im}\left(r_{\sigma}\right) \frac{\mathrm{e}^{-2 \kappa z}}{\kappa},
\end{aligned}
$$

Close to the threshold ( $v-1 / n$ becomes small) the range in $k_{x}$ becomes narrow $(c=1)$ :

$$
n \omega-\frac{\omega}{v}=\frac{\omega}{v}(n v-1) \approx n \omega(n v-1) \text {. }
$$

For the range in $k_{y}$ we find:

$$
\left|k_{y}\right| \leq \sqrt{(n \omega)^{2}-k_{x}^{2}}=\sqrt{\left(n \omega-k_{x}\right)\left(n \omega+k_{x}\right)} \leq \omega \sqrt{(2 n / v)(n v-1)} .
$$

Hence the wave vectors in the reflection coefficients become

$$
\begin{aligned}
& n^{2} \omega^{2}-\frac{\omega^{2}\left(n^{2} v^{2}-1\right)}{v^{2}} \leq k_{\|}^{2} \leq n^{2} \omega^{2}, \\
& \left(n^{2}-1\right) \omega^{2}-\frac{\omega^{2}\left(n^{2} v^{2}-1\right)}{v^{2}} \leq \kappa^{2} \leq\left(n^{2}-1\right) \omega^{2}, \\
& 0 \leq \kappa_{n}^{2} \leq \frac{\omega^{2}\left(n^{2} v^{2}-1\right)}{v^{2}} .
\end{aligned}
$$

For small values of $\kappa_{n}$ the reflection coefficients thus scale like $\operatorname{Im} r_{\sigma} \sim \sqrt{n v-1}$. For the integration domain in frequency, we distinguish whether the particle is lossy or lossless:

$$
\begin{aligned}
\text { lossy: } & 0 \leq \omega<\mathcal{O}\left[1 /\left(z \sqrt{n^{2}-1}\right)\right]<\infty, \\
\text { no loss: } & \omega_{\mathrm{a}}=\frac{\Omega / \gamma}{n v-1} \leq \omega<\mathcal{O}\left[1 /\left(z \sqrt{n^{2}-1}\right)\right]<\infty
\end{aligned}
$$

where the upper limit arises from the exponential $\mathrm{e}^{-2 \kappa z}$. For the comoving frequency $\omega^{\prime}=$ $\gamma\left(\omega-v k_{x}\right)$ we find:

$$
\begin{aligned}
\text { lossy: } & 0 \leq-\omega^{\prime} \leq \gamma \omega(n v-1)<\mathcal{O}\left[\gamma(n v-1) /\left(z \sqrt{n^{2}-1}\right)\right], \\
\text { no loss: } & -\omega^{\prime}=\Omega .
\end{aligned}
$$

Hence we see how the relevant frequency ranges are separated by a considerable margin between the lossy and the lossless case. This suggests that we can capture both from two different approximations. Note that the lossy case is dominated by a range of quasi-static frequencies which becomes narrower, the closer the velocity gets to the Cherenkov threshold (difference $v-1 / n$ ). 


\section{Particle with no losses}

In the case of no losses we approximate the particle's resonance with a $\delta$-distribution. Formally this is done by taking the limit $1 / \tau \rightarrow 0$ in Eq.(17). Because we only have to focus on $\omega<0$ we find [see also Eq.(18)]:

$$
\left(4 \pi a^{3}\right) \operatorname{Im} \frac{\Omega^{2}}{\Omega^{2}-\omega^{2}-\mathrm{i} \omega / \tau} \approx-\left(4 \pi a^{3}\right) \frac{\pi}{2} \delta(\omega+\Omega) .
$$

The oscillator strength $\operatorname{Im} \alpha\left(\omega^{\prime}\right)$ thus fixes the $k_{x}$-wave vector to (label 'a' from 'apex' of hyperbola)

$$
\text { no loss: } \quad k_{x}=k_{x \mathrm{a}}=\frac{\omega+\Omega / \gamma}{v}, \quad \omega>\omega_{\mathrm{a}}=\frac{\Omega / \gamma}{n v-1} .
$$

Note that $\omega$ becomes 'large' near the threshold $n v=1$. The expressions for wave vectors $\kappa$ and $\kappa_{n}$ read

$$
\begin{aligned}
k_{y \max }^{2} & =(n \omega)^{2}-k_{x \mathrm{a}}^{2} \\
\kappa_{n}^{2} & =k_{y \max }^{2}-k_{y}^{2} \\
\kappa & \approx \sqrt{n^{2}-1} \omega-\frac{k_{y \max }^{2}-k_{y}^{2}}{2 \sqrt{n^{2}-1} \omega} .
\end{aligned}
$$

For the reflection coefficients this yields the approximation (keeping only the lowest order in $\kappa$ )

$$
\operatorname{Im} r_{p} \approx \frac{2}{n^{2} \sqrt{n^{2}-1}} \frac{k_{y \max }}{\omega}\left(1-q_{y}^{2}\right)^{1 / 2}
$$

where $0 \leq q_{y} \leq 1$ is a scaled version of $k_{y}=q_{y} k_{y \max }$. For the exponential, we include the next order of $\kappa$ and take

$$
\frac{\mathrm{e}^{-2 \kappa z}}{\kappa} \approx \frac{\mathrm{e}^{-2 \omega z \sqrt{n^{2}-1}}}{\omega \sqrt{n^{2}-1}}\left[1+\frac{z k_{y \max }^{2}}{\sqrt{n^{2}-1} \omega}\left(1-q_{y}^{2}\right)\right] .
$$

We find that the p-polarization yields the leading contribution and use the lowest-order approximation

$$
\begin{aligned}
& \phi_{p}\left(\omega, \mathbf{k}_{\|}\right)=2 \omega^{2}\left(n^{2}-1\right)+\mathcal{O}\left[\omega^{2}(n v-1)\right] \\
& \phi_{s}\left(\omega, \mathbf{k}_{\|}\right)=\mathcal{O}\left[\omega^{2}(n v-1)\right] .
\end{aligned}
$$


Putting everything together in the leading order, we get the approximate expression

$$
\begin{aligned}
F_{x} \approx-\frac{4 \hbar\left(4 \pi a^{3}\right)}{(2 \pi)^{3}} \frac{2 \pi \Omega}{n v \gamma^{2}} \int_{\omega_{\mathrm{a}}}^{\infty} \mathrm{d} \omega \omega k_{y \max }^{2} \mathrm{e}^{-2 \omega z \sqrt{n^{2}-1}} \\
\quad \times \int_{0}^{1} \mathrm{~d} q_{y}\left(1-q_{y}^{2}\right)^{1 / 2}\left[1+\frac{z k_{y \max }^{2}}{\omega \sqrt{n^{2}-1}}\left(1-q_{y}^{2}\right)\right] .
\end{aligned}
$$

A convenient formulation for

$$
k_{y \max }^{2}=(n v-1) \frac{\omega-\omega_{\mathrm{a}}}{v^{2}}\left[2 \omega+(n v-1)\left(\omega+\omega_{\mathrm{a}}\right)\right]
$$

shows the scaling above threshold. The $q_{y}$-integral can be performed and produces Eq. (23).

\section{Particle with losses}

As outlined in the estimates above, we can use the low-frequency approximation

$$
\omega^{\prime} \ll \Omega: \quad \operatorname{Im} \alpha\left(\omega^{\prime}\right) \approx 4 \pi a^{3} \frac{\omega^{\prime}}{\Omega^{2} \tau} .
$$

for the polarizability. This assumes that the frequency range for $\omega^{\prime}$ near zero is sufficient to capture the integral and ignores the resonant peak. The integrals that must be performed are

$$
\begin{aligned}
& F_{x} \approx-\frac{4 \hbar\left(4 \pi a^{3}\right)}{(2 \pi)^{3}} \frac{4 n^{2}}{\Omega^{2} \tau} \int_{0}^{\infty} \mathrm{d} \omega \omega^{5} \mathrm{e}^{-2 \omega z \sqrt{n^{2}-1}} \\
& \int_{1 /(n v)}^{1} \mathrm{~d} q_{x} q_{x}\left(1-q_{x}^{2}\right)\left(v n q_{x}-1\right) \\
& \int_{0}^{1} \mathrm{~d} q_{y} \sqrt{1-q_{y}^{2}}\left[1+\frac{\omega z n^{2}\left(1-q_{x}^{2}\right)}{\sqrt{n^{2}-1}}\left(1-q_{y}^{2}\right)\right] .
\end{aligned}
$$

The $q_{y}$-integral is the same as before [Eq. 23 ] ] and gives

$$
\begin{aligned}
F_{x} \approx-\frac{4 \hbar\left(4 \pi a^{3}\right)}{(2 \pi)^{3}} \frac{4 n^{2}}{\Omega^{2} \tau} \int_{0}^{\infty} \mathrm{d} \omega \omega^{5} \mathrm{e}^{-2 \omega z \sqrt{n^{2}-1}} & \\
& \int_{1 /(n v)}^{1} \mathrm{~d} q_{x} q_{x}\left(1-q_{x}^{2}\right)\left(v n q_{x}-1\right) \frac{\pi}{4}\left(1+\frac{3 \omega z n^{2}\left(1-q_{x}^{2}\right)}{4 \sqrt{n^{2}-1}}\right) .
\end{aligned}
$$

The $q_{x}$-integral yields the force spectrum in eq. 25. 


\section{References}

[1] E.V. Teodorovich. On the contribution of macroscopic Van Der Waals interactions to frictional force. Proc. Roy. Soc. (London) A, 362:71-77, 1978.

[2] L.S. Levitov. Van der Waals' friction. Europhys. Lett., 8:499, (1989).

[3] V. G. Polevoi. Tangential molecular forces caused between moving bodies by a fluctuating electromagnetic field. Sov. Phys. JETP, 71(6):1119-24, 1990.

[4] J.B. Pendry. Shearing the vacuum-quantum friction. J. Phys.: Cond. Matt., 9:10301, 1997.

[5] T.G. Philbin and U. Leonhardt. No quantum friction between uniformly moving plates. New J. Phys., 11:033035, 2009.

[6] Mehran Kardar and Ramin Golestanian. The "friction" of vacuum, and other fluctuationinduced forces. Rev. Mod. Phys., 71(4):1233-45, 1999.

[7] P. C. W. Davies. Quantum vacuum friction. J. Opt. B: Quant. Semiclass. Opt., 7(3):S40, 2005.

[8] A.I. Volokitin and B.N.J. Persson. Near-field radiative heat transfer and noncontact friction. Rev. Mod. Phys., 79(4):1291-1329, 2007.

[9] Mohammad F. Maghrebi, Ramin Golestanian, and Mehran Kardar. Quantum Cherenkov radiation and non-contact friction. Phys. Rev. A, 88:042509, 2013.

[10] W L Schaich and J Harris. Dynamic corrections to Van der Waals potentials. J. Phys. F: Metal Phys., 11(1):65-78, 1981.

[11] G.V. Dedkov and A.A. Kyasov. Electromagnetic friction forces on the scanning probe asperity moving near surface. Phys. Lett. A, 259(1):38-42, 1999.

[12] Stefan Scheel and Stefan Yoshi Buhmann. Casimir-Polder forces on moving atoms. Phys. Rev. A, 80(4):042902, 2009.

[13] G. Barton. On van der Waals friction. II: Between atom and half-space. New J. Phys., $12: 113045,2010$. 
[14] A.A. Kyasov and G.V. Dedkov. Relativistic theory of fluctuating electromagnetic slowing down of neutral spherical particles moving in close vicinity to a flat surface. Nucl. Instr. Meth. Phys. Res. B, 195(3-4):247-58, 2002.

[15] G.V. Dedkov and A.A. Kyasov. Fluctuation-electromagnetic interaction of a moving neutral particle with a condensed-medium surface: relativistic approach. Phys. Sol. State, 51(1):126, 2009.

[16] Gregor Pieplow and Carsten Henkel. Fully covariant radiation force on a polarizable particle. New J. Phys., 15(2):023027, 2013.

[17] Diego A. R. Dalvit, Paulo A. Maia Neto, and Francisco Diego Mazzitelli. Fluctuations, dissipation and the dynamical Casimir effect, volume 834 of Lecture Notes in Physics, chapter 13, pages 419-57. Springer, Berlin Heidelberg, 2011.

[18] B.M. Bolotovskii. The theory of the Vavilov-Cherenkov effect. Uspekhi Fiz. Nauk, 62:20146, 1957. (in Russian); see also Sov. Phys. Uspekhi 4 (1962) 781-811.

[19] Sergei M. Rytov, Yurii A. Kravtsov, and Valeryan I. Tatarskii. Elements of Random Fields, volume 3 of Principles of Statistical Radiophysics. Springer, Berlin, 1989.

[20] P A Cherenkov. Visible radiation produced by electrons moving in a medium with velocities exceeding that of light. Phys. Rev., 52(4):378, 1937.

[21] I.E. Tamm and I.M. Frank. Coherent radiation of fast electrons in a medium. Dokl. Akad. Nauk SSSR, 14(3):107-12, 1937.

[22] C. K. Carnaglia and L. Mandel. Quantization of evanescent electromagnetic waves. Phys. Rev. D, 3:280-96, 1971.

[23] C. Eberlein and D. Robaschik. Quantum electrodynamics near a dielectric half-space. Phys. Rev. D, 73(2):025009, 2006.

[24] V.E. Mkrtchian. Interaction between moving macroscopic bodies: viscosity of the electromagnetic vacuum. Phys. Lett. A, 207(5):299-302, 1995.

[25] V.P. Frolov and V.L. Ginzburg. Excitation and radiation of an accelerated detector and anomalous Doppler effect. Phys. Lett. A, 116(9):423-426, 1986. 
[26] Mario G. Silveirinha. Quantization of the electromagnetic field in non-dispersive polarizable moving media above the Cherenkov threshold. Phys. Rev. A, 88:043846, 2013.

[27] R. Zhao, A. Manjavacas, F. J. García de Abajo, and J. B. Pendry. Rotational quantum friction. Phys. Rev. Lett., 109:123604, 2012.

[28] H. Hövel, S. Fritz, A. Hilger, U. Kreibig, and M. Vollmer. Width of cluster plasmon resonances: Bulk dielectric functions and chemical interface damping. Phys. Rev. B, 48(24):18178-88, 1993.

[29] Uwe Kreibig and Michael Vollmer. Optical Properties of Metal Clusters, volume 25 of Springer Series in Materials Science. Springer, Berlin Heidelberg New York, 1995.

[30] Stéphane Berciaud, Laurent Cognet, Philippe Tamarat, and Brahim Lounis. Observation of intrinsic size effects in the optical response of individual gold nanoparticles. Nano Lett., 5(3):515-18, 2005.

[31] Lucía B Scaffardi and Jorge O Tocho. Size dependence of refractive index of gold nanoparticles. Nanotechnology, 17(5):1309-15, 2006.

[32] Vitalii L Ginzburg. Radiation by uniformly moving sources (Vavilov-Cherenkov effect, transition radiation, and other phenomena). Phys. Uspekhi, 39(10):973-82, 1996.

[33] F. Intravaia, R. O. Behunin, and Diego A. R. Dalvit. Quantum friction and non-equilibrium fluctuation theorems. arXiv:1308.0712, comment by P. Milonni, arXiv:1309.1490, 2013.

[34] J.S. Høye and I. Brevik. Casimir friction force between polarizable media. Eur. Phys. J. D, 66(6):1-5, 2012. 